\title{
Anesthesiological Management of Patients Operated on Urgently in a Structure on the Outskirts of Bamako (Mali)
}

Coulibaly Théodore Habib Maxime ${ }^{1 *}$, Dramé Ahmadou Ibrahim ${ }^{2}$, Timbiné Kalba ${ }^{2}$, Bengaly Mamadou ${ }^{1}$ Tall Fadima ${ }^{1}$, Diani Nouhoum ${ }^{2}$

\footnotetext{
${ }^{1}$ Intensive care unit of the Hospital center of kati-Mali

${ }^{1,2}$ Neurosurgical Intensive, Care of the Hospital of Mali
}

DOI: $10.36347 /$ sasjs.2020.v06i12.004

| Received: 29.11.2020 | Accepted: 14.12.2020 | Published: 18.12 .2020

*Corresponding author: Coulibaly Théodore Habib Maxime

Abstract

In this prospective study spread over a period of 12 months, the objective was to describe emergency Anesthesiological management and possible incidents and accidents. Surgical, neurosurgical, gynecological and orthotrauma emergencies were concerned by this study. 59 patients were collected during the study period. The 15-30 age group was the most prevalent and the sex ratio was 1.36 in favor of males. The majority of patients $(61 \%)$ were in good general condition and the majority of our patients had no history. General anesthesia was the most widely used anesthetic protocol. Acute appendicitis was the most common pathology encountered both preoperatively and intraoperatively. Seventy-two point nine $(72.9 \%)$ of interventions were completed in less than 90 minutes. Intraoperative antibiotic prophylaxis was performed in $86.4 \%$ of cases. The most common side effect was low blood pressure. The outcome was favorable in $89.8 \%$ of cases and 6 deaths were observed. Urgent intervention by the anesthesiologist is necessary for life-threatening reasons.

Keywords: Anesthesiology, emergencies, incidents, accidents, antibiotic prophylaxis.

Copyright $\odot 2020$ The Author(s): This is an open-access article distributed under the terms of the Creative Commons Attribution 4.0 International License (CC BY-NC 4.0) which permits unrestricted use, distribution, and reproduction in any medium for non-commercial use provided the original author and source are credited.

\section{INTRODUCTION}

Emergency is the pressing condition of a patient whose treatment cannot be postponed without endangering his life [1]. Current or imminent abnormal situation requiring rapid intervention or special regulations targeting persons or property in order to preserve the health, safety or well-being of persons or to limit damage to documents or property [2]. The difficulties associated with an emergency anesthesia differ from one patient to another. Each situation is distinguished by the type of patient and the importance of the impact of the impact of the disease on vital functions. However, two characteristics are almost always found: the urgency of the intervention and the urgency of the initiation of intensive care. The difficulties linked to surgical emergencies remain more topical than ever in our country, their prognosis still remains severe with a mortality of 20 to $30 \%$ [3]. The aim of our study was to analyze the anesthesiology management of patients operated on in emergency rooms.

\section{METHODOLOGY}

It was a prospective and descriptive study spread over a period of 12 months (January 1 to
December 31, 2009) and carried out at the level of the of the anesthesia and intensive care unit of the hospital of Kati. It concerned all patients admitted to hospital and having undergone a pre-anesthetic visit for surgery during the study period. Patients who were anesthetized outside the emergency setting were not included in our study. Our data were collected on the basis of anesthesia files and postoperative care files. The variables measured were: socio-demographic data, clinical and para-clinical data, quantification of anesthesia, anesthetic protocol and drugs used, intraoperative blood transfusion, surgical technique, duration of the intervention, undesirable effects encountered, their nature, their time of onset and their evolution, postoperative management. SPSS 12.0 software was used for data entry and analysis.

\section{RESULT}

During the period of our study, 59 patients were seen for a pre-anesthetic visit, including 34 $(57,60)$ men and 25 women $(42,40)$. The $15-30$ age group was the most represented $(45,8 \%)$. On examination, the majority of patients were in good general condition $(61 \%)$. Abdominal surgical emergencies were the most common $(72,90 \%)$ followed 
by trauma $(22 \%)$. Acute appendicitis was the most observed preoperative diagnosis $(23,7)$ followed by bowel obstruction $(16,90)$. Seventeen $(17 \%)$ of the patients had a history of anesthesiology and twenty-two $(22 \%)$ of the patients had a medical history. The group/Rhesus and the N.F.S were the most urgent para clinical examinations (Table-1).

Table-1: Distribution of patients according to the para clinical examinations actually carried out

\begin{tabular}{|l|l|l|l|}
\hline Examinations & OUI & NON & Total \\
\hline Group/Rhesus & $61 \%$ & $9 \%$ & $100 \%$ \\
\hline Blood count & $61 \%$ & $9 \%$ & $100 \%$ \\
\hline Rx standard & $22 \%$ & $78 \%$ & $100 \%$ \\
\hline Serum creatinine & $15 \%$ & $5 \%$ & $100 \%$ \\
\hline Glycemia & $14 \%$ & $6 \%$ & $100 \%$ \\
\hline Ultrasound & $12 \%$ & $88 \%$ & $100 \%$ \\
\hline Scanner & $\%$ & $98 \%$ & $100 \%$ \\
\hline
\end{tabular}

General anesthesia was performed in $525 \%$ of cases (Table-2).

Table-2: Distribution of patients according to the technical anesthesia.

\begin{tabular}{|l|l|l|}
\hline Anesthesia technique & Frequency & Percentage \\
\hline General anaesthesia & 31 & 52,5 \\
\hline Rachianesthesia & 26 & 44,1 \\
\hline Trunk block & 1 & 1,7 \\
\hline A. Local & 1 & 1,7 \\
\hline Total & 59 & 100 \\
\hline
\end{tabular}

Bupiyacaine 0,5 alone was the most widely used drug in ALR (RA) (81,50\%). Ketamine and succinycholine were the most widely used maintenance drugs. IOT with manual ventilation was the most performed ventilation mode $(96,60 \%)$. The anesthetic act was performed in $33,9 \%$ of cases under the responsibility of a doctor (Table-3).

Table-3: Distribution according to the qualification of the anesthesia

\begin{tabular}{|c|c|l|}
\hline Qualification & Frequency & Percentage \\
\hline Doctor $+\mathrm{PhD}$ student & 3 & 5,10 \\
\hline Doctor + Anesthesia technicians & 17 & 28,80 \\
\hline Anesthesia technicians + PhD student & 23 & 39,00 \\
\hline Anesthesia technicians & 16 & 27,10 \\
\hline Total & 59 & 100,00 \\
\hline
\end{tabular}

Antibiotic prophylaxis was performed in $86,4 \%$ of cases. The transfusion was performed only once. General anesthesia was the most common source of adverse events (Table-4) and arterial hypotension was the most common adverse event $(23,70 \%)$.

Table-4: Distribution of adverse events according to type of anesthesia

\begin{tabular}{|l|l|l|l|l|l|l|}
\hline Effective & \multicolumn{3}{l|}{ Types of anesthesia } & \multirow{2}{*}{ Total } \\
\hline Types of events & & A.G & R.A & A. L & B. Trunk & \\
\hline \multirow{5}{*}{} & Hypotension & 5 & 9 & 0 & 0 & 14 \\
\cline { 2 - 7 } & Hypertension & 1 & 1 & 0 & 0 & 2 \\
\cline { 2 - 7 } & Tachycardia & 2 & 0 & 0 & 0 & 2 \\
\cline { 2 - 7 } & Hypotension-tachycardia & 2 & 0 & 0 & 0 & 2 \\
\cline { 2 - 7 } & Puncture of the balloon catheter & 1 & 0 & 0 & 0 & 1 \\
\cline { 2 - 7 } & Hypotension-Agitation-Prolonged awakening & 1 & 0 & 0 & 0 & 1 \\
\cline { 2 - 7 } & Hypotension-bradycardia & 0 & 1 & 0 & 0 & 1 \\
\cline { 2 - 7 } & None & 19 & 15 & 1 & 1 & 36 \\
\hline Total & & 31 & 26 & 1 & 1 & 59 \\
\hline
\end{tabular}

The majority of adverse events occurred preoperatively (32,20\%). The majority of the interventions took place in less than 90 minutes $(73,90 \%)$. In the majority of cases paracetamol infusion alone was the analgesic used $(62,70 \%)$. The outcome was favorable in $89,8 \%$ of cases (Table-5) and only $30,50 \%$ of the patients had undergone intensive care.

Table-5: Distribution of patients according to the evolution

\begin{tabular}{|l|l|l|}
\hline Evolution & Frequency & Percentage \\
\hline Favorable & 53 & 89,8 \\
\hline Death & 6 & 10,2 \\
\hline Total & 59 & 100 \\
\hline
\end{tabular}




\section{DISCUSSION}

In our study, the 15-30 age group was the most represented $(45,8 \%)$. This observation could be explained by the structure of the African population, which is predominantly young. Our result was close to those of Ouattara K. [4] wih $41,10 \%$ between $15-29$ years and of Christian S. [5] with $42 \%$. In our study, there was a male predominance $(57,6 \%)$ against $42 \%$ of women. Our result was comparable to that of Ouattara K. [4] who found male predominance with $71,76 \%$. Tiogo [6] on the contrary before found a sex ratio of 1,29 in favor of the female sex. This observation was different from ours. Abdominal surgical emergencies were the most common $(72,90 \%)$ followed by trauma $(22 \%)$. Acute appendicitis was the most observed preoperative diagnosis $(23,7 \%)$ followed by intestinal obstruction (16.90). The medical history was found in $22 \%$ of our patients. Our result was close to those of Fotso K [7] and Ouattara K [4] with respectively $17,22 \%$ and $19,9 \%$ but far behind that of Moussa K [8] with $44 \%$. Anesthesia history was found in $17 \%$ of patients and general anesthesia was the most common anesthetic history with $10,2 \%$. Our result was different from that of Ouattara K [4] with 3,5\% anesthetic history and the most frequent was lororegional anesthesia. In our study the blood group/rhesus and the blood count were the only examinations performed (61\%) each and the intervention was started most often without waiting for the results of these examinations. This observation could be explained by the unavailability of other emergency examinations in the structure but also by the lack of qualified personal to carry out these examinations. General anesthesia was the most performed during our study $(52,5 \%)$. The same is true for Ouattara K [4] with 97,3\%. This choice seems justified if one takes into account the particular working conditions in an African environment. In the authors agree in emphasizing the risks associated with locoregional peri-medullary anesthesia during emergencies, in particular because of the hypovolimic terrain. Only one case of peripheral ALR was performed. This observation could be explained by the absence of suitable material for the realization of this technique and also, the lack of mastery of the technique. For the induction of GA the most used hypnotics were Ketamine followed by propofol. This observation was different from those of ASSINA Sau [12] and Ouattara K [4] who had found thiopental after Ketamine. Succinylcholine was the cuare of choice during induction and fentanyl was the only pain reliever used for GA the same observation to Ouattara K [4]. Locoregional anesthesia (RA) was done by bupiyacaine alone $(81,5 \%)$ and by bupiyacaine associated with Fentanyl $(3,7 \%)$. Our results were comparable to that of Bengaly M [13] who had found for bupiyacaine alone $(81,4 \%)$ and for bupiyacaine associated with Fentanyl $(15,1 \%)$. Orotracheal intubation associated with manual ventilation was the ventilation mode used in more than half of cases $(96,6 \%)$. Our result far exceeded that of Ouattara K [4] who found $51,08 \%$ for the same ventilation mode. The main reason being insufficient ventilator, antibiotic prophylaxis was performed in more than half of the cases. In our study, the majority of anesthetic acts were performed by anesthesia technicians and sometimes with the help of $\mathrm{PhD}$ students $(66,1 \%)$. This finding could be explained by the insufficient number of specialist physicians in the field who can provide both day and night coverage. Our result was not comparable to those of the SFAR [14]; by Venet. C [15] and of Gravot. B [16] who report $100 \%$ responsibility of an anesthesiologist due to the sufficient number of anesthesiologist due to the sufficient number of anesthesiologists their respective country. General anesthesia was the most common source of adverse events and arterial hypotension was the most common adverse event $(23,70 \%)$. The majority of adverse events occurred preoperatively $(32,20 \%)$. This finding was identical to that of Bengaly $M$ [13], but different from that of Tiogo [6] in whom the adverse events occurred during induction. Paracetamol alone as an infusion was the analgesic used for the management of postoperative pain. This finding could be explained by the absence of a postoperative pain management protocol but also by poor knowledge of pain management by the nursing staff. In our study $72,9 \%$ of the interventions took place in less than 90 minutes. Our results were comparable to those of Christian S [5] and Ouattara K [4] with respectively $69, \%$ and $73,1 \%$. The outcome was favorable in $89,8 \%$ of cases. However $10,2 \%$ of patients died postoperatively. These deaths could be explained by the delay and the poor quality of the care. Our result was different from those of the literature [5, 17] which found $8,87 \%$ and $0,6 \%$ of preoperative deaths, respectively.

\section{CONCLUSION}

The management of emergency patients with a view to surgery is a major challenge for any anesthetistresuscitator. The success of such an intervention requires good preparation. Particular emphasis should be placed on the management of postoperative pain.

\section{REFERENCES}

1. Doumbia D. Study of surgical emergencies received at Point G hospital from November 1981 to November 1982. Thesis: Med : University of Bamako. 1982.

2. Edicef 58, $3^{\text {ème }}$ édition, rue Jean-bleuzen, F 92178 Vanves cedex.

3. Camara S. Anesthesia and intensive care problem psed by emergency abdominal surgery to the hospital of Gabriel Touré. Thesis: Med: University of Bamako.

4. Ouattara K. Emergency medication and anesthetic techniques at the hospital Gabriel Touré about 408 cases. Thesis: Med: University of Bamako. 2008.

5. Christian Serge T. Activities of the anesthesia department at the Gabriel Toure university 
hospital: 2004 review. Thesis: Med: University of Bamako. 2006.

6. Tiogo C. Incident and accident related to anesthesia in Yaoundé. Epidemiological study and preventive aspects. Thesis: Med: University of Yaoundé1. 1997.

7. Fotso K, Gilles M. Incident and accident of anesthesia at Point $\mathrm{G}$ hospital. Epidemiological, clinical and prognostic profile. Thesis: Med: University of Bamako. 2005.

8. Konate M. Difficult tracheal intubation in thyroid surgery in the anesthesia and intensive care unit of Point G hospital Thesis: Med: University of Bamako. 2006.

9. Bakara A, Louis F, Noueid. Awareness following different technics of anaesthesia of caesarean. J Anesthesiology. 1989; 645-648.

10. Fernandez M, Edouard D. Obstetrical emergencies (éd Masson, Paris). 1996; 951-73.

11. Haimeur C, Tazi A, Alaoui I. Caesarean anesthesia: comparison between thiopental and ketamine. Cahier-aneth. 1997; 9-13.
12. Assina. S. Practice of anesthesia in Chad in 2001 : Curent situation and prospect for improvement. Thesis: Med: University of Cotonou. 2001.

13. Bengaly. M. Anesthetic activities at Kati hospital : 12 months assessment. Thesis : Med: University of Bamako. 2008.

14. SFAR. The practice of anesthesia in France in 1996. Ann Fr Anesthesia Intensive Care. 1997; (17): 1299- 301.

15. Venet C. Collection of anesthesia incidents and accidents at the Grenoble University Hospital. Thesis: Med: University of Grenoble. 2000.

16. Gravot B. Events, incidents and accidents related to anesthesia, analysis of one year of anesthesiology activity in urban hospitals in Nancy. Thesis: Med: University of Nancy. 1995.

17. Dicko ME. Anesthetic risk in scheduled surgery at the hospital of Gbriel Touré. Thesis: Med: University of Bamako. 1990. 